\title{
ESSAI
}

Invité article

DOI: http://dx.doi.org/10.1590/So034-759020150212

\section{LE CHAMP DES ÉTUDES ORGANISATIONNELLES : LE REGARD CRITIQUE D'UN CHERCHEUR PLURILINGUE}

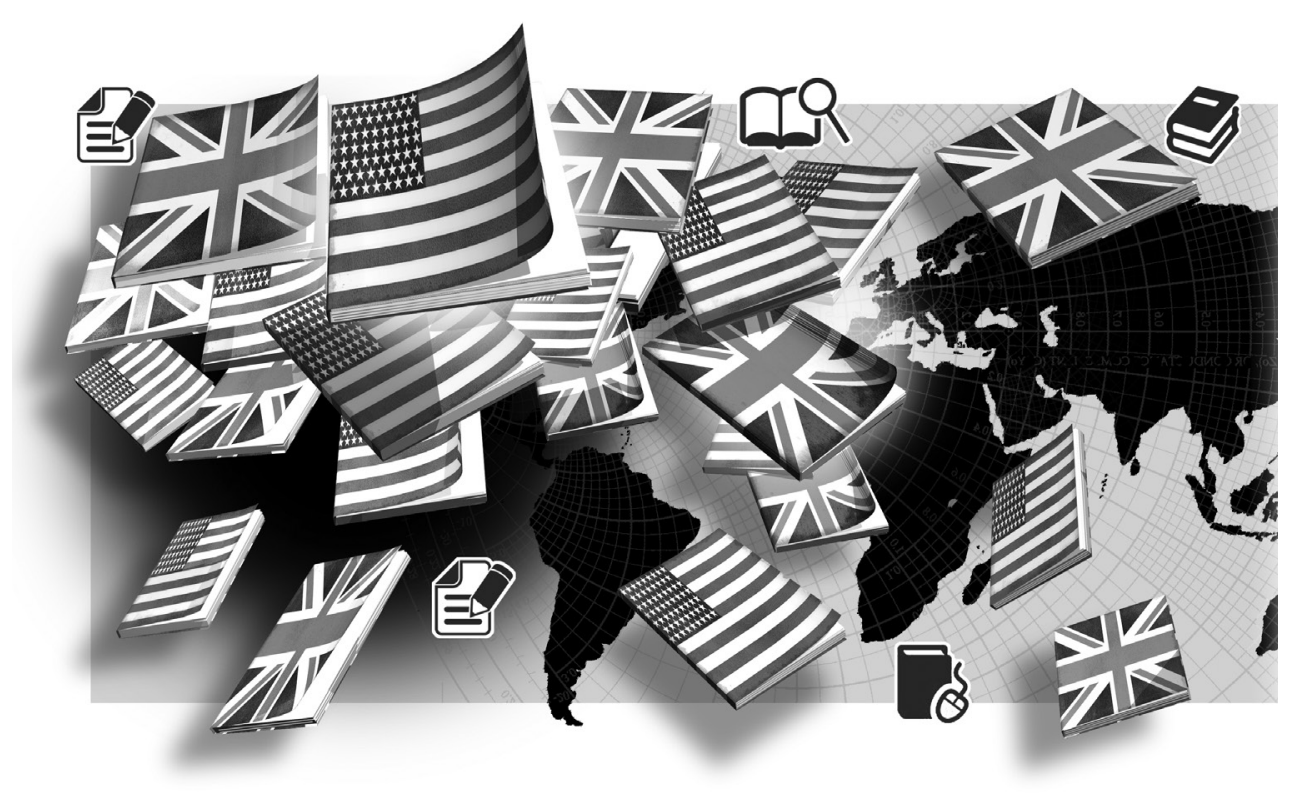

Le numéro spécial dont j'ai l'honneur de faire le mot d'introduction a pour objet de questionner à partir des pays du Sud l'hégémonie qu'exerce la pensée anglo-saxonne largement dominante dans le champ du management et des études organisationnelles. J'utilise ici volontairement d'entrée le concept de champ proposé par Bourdieu $(1982,1987)$ car il nous permet d'affirmer sans le moindre doute que le champ dont il est question dans ce numéro, est un champ social, c'est-à-dire un espace structuré, hiérarchisé d'acteurs et de positions, qui se définit par des enjeux et des intérêts spécifiques mobilisant des formes de capital variées (économique, culturel, social et symbolique). Ce champ nécessite également un système de dispositions approprié par les acteurs, ce que Bourdieu $(1982,1987)$ désigne par habitus, un système permettant à ses acteurs de faire face aux règles du jeu en vigueur en son sein, et d'y occuper ainsi une place (Bourdieu, 1982, 1987) ; le champ de la recherche en gestion et des études organisationnelles est bel et bien un champ de ce type.

Fruit d'un appel à communication rédigée par Ernesto Gantman, Hèla Yousfi et Rafael Alcadipani, la lecture des articles sélectionnés, dont je partage très largement les constats et les analyses, m’a inspiré une série de réflexions.

La première vient rappeler que le champ de la recherche en gestion et des études organisationnelles fait partie du champ des sciences sociales et qu'en tant que champ spécifique, il est en ef-

JEAN-FRANÇOIS CHANLAT jean-francois.chanlat@dauphine.fr Professeur et co-responsable scientifique de la Dauphine Université Paris, Chaire Management et Diversité - Paris, France fet marqué par la domination qu'exerce le champ anglo-américain et les normes que celui-ci met en place (Dameron \& Durand, 2008). Le poids de la production américaine est, nous le savons tous, historiquement considérable, même s’il a existé et s'il existe toujours des pensées riches dans ce domaine dans d'autres parties du monde dont certains articles de ce numéro témoignent (Alcadipani \& Rosa, 2011 ; Ibarra-Colado, 2006 ; Bayley \& Clegg, 2007 ; Pesqueux \& Tyberghien, 2009 ; Batti- 
lana, Anteby, \& Sengul, 2010 ; Courpasson, Arellano-Gault, Brown, \& Lounsbury, 2008 ; Chanlat, 1994 ; 2014a).

Par conséquent, comme l'ont remarqué de nombreux observateurs, et les auteurs des articles présentés ici, selon les lieux où le chercheur se trouve, sa production sera plus ou moins prise en compte par le champ dominant nord-américain (Bargiela-Chiappini, 2001 ; Tsui, 2007 ; Adler \& Harzing, 2009 ; Battilana, Anteby \& Sengul, 2010 ; Courpasson et alii., 2008 ; Chanlat, 2014b). C'est ainsi que, pour Üsdiken (2010), le champ de la recherche en gestion se divise entre trois espaces : le cœur qu'il associe aux États-Unis, ceux-ci étant depuis la fin de la Seconde Guerre mondiale la source la plus influente en matière d'idées, un second centre (le Royaume-Uni), une semi périphérie (l'Europe du Nord et de l'Ouest) et une périphérie (l'Europe du Sud et de l'Est à laquelle nous pourrions ajouter les pays en développement ou émergents - ce qu'Üsdiken ne fait pas puisque son article porte essentiellement sur l'univers euro-américain mais ce que font de manière centrale les différents auteurs de ce numéro.

La seconde réflexion porte sur la notion de résistance à cette hégémonie. Cette résistance prend différentes formes. Comme le montrent, là encore, les auteurs de ce numéro spécial, il existe des résistances qui mêlent à la fois des éléments politiques, socioculturels, linguistiques et intellectuels; en effet, de nombreux champs linguistiques se distinguent du champ américain par leurs choix épistémologiques et sociaux et par la défense d'une production locale, nationale et régionale. Si cette tendance est particulièrement observable dans le champ francophone dont l'autonomie est relativement importante par rapport au champ dominant (Chanlat, 2014), on peut également l'observer également dans le champ de langue anglaise d'influence britannique et européenne (Grey, 2010 ; Willmott, 2011). Mais il reste que le mimétisme demeure important dans certains champs régionaux, lesquels doivent composer avec la mondialisation croissante de l'enseignement supérieur (Lussier, 2014).

Le rôle joué par les systèmes de classement de revues (et surtout l'importance qui leur est accordée), et plus généralement celui joué par les critères d'évaluation des productions intellectuelles, ne sont pas sans lien avec cet alignement systématique sur la production anglo-américaine (Lussier, 2014). Car si on observe une résistance dans les pays dont la langue n'est pas l'anglais, on peut remarquer cependant que les revues les plus cotées dans de nombreux champs nationaux sont systématiquement des revues de langue anglaise, pour ne pas dire américaine. Dans un tel contexte, il devient donc quasiment impossible, pour un chercheur non anglophone, de participer au débat, s’il ne maîtrise pas parfaitement la langue anglaise (Tietze, 2004). L'établissement de ces classements devient donc un enjeu clé pour les acteurs concernés, notamment pour ceux qui ne sont pas de langue anglaise (Berry, 2004 ; Eraly, 2011 ; Hatchuel, 2004 ; Tsuda, 2013 ; Aquino-Alves \& Pozzebon, 2013 ; Chanlat, 2014a).

La troisième concerne les effets de cette hégémonie anglo-américaine. Si ceux-ci ont été abordés par de nombreux chercheurs déjà cités, et rappelés ici, chacun à leur manière, par les auteurs de ce numéro spécial, moins nombreux sont ceux qui se sont intéressés aux effets cognitifs et au processus de production intellectuel de rédiger en anglais en tant que tels. S'ils l'ont été, c'est surtout par des chercheurs non anglophones. Le Norvégien Ljosland (2007) rappelle, par exemple, que dans son pays la langue dans laquelle est rédigée une thèse d'économie est dans $82 \%$ des cas, l'anglais, dans $16 \%$ des cas le norvégien, et les $2 \%$ restants une rédaction dans ces deux langues. Cette anglicisation massive produit, selon lui, une “colonisation mentale" et conduit à une perte de sujets de recherche potentielle- ment intéressants pour la société norvégienne (Ljosland, 2007). Il est intéressant de constater que cette situation est aujourd'hui observable dans certaines institutions du monde non anglophone, notamment latines, particulièrement tournées vers la reconnaissance apportée par le champ anglo-américain. L'article de notre collègue de Madrid est assez exemplaire à cet égard.

D’autres collègues ont également observé ce qu'ils appellent une fermeture symbolique. Lors de travaux réalisés conjointement par des Finlandais et des Britanniques, les chercheurs finlandais concernés ont fait le constat que la vision finlandaise et les mots en finlandais étaient subordonnés à la signification anglaise (Meriläinen, Tienari, Thomas, \& Davies, 2008), voire remplacés par des mots anglais, et ce ne fut que par une résistance et un questionnement de ces processus que les chercheurs finlandais sont parvenus à se faire respecter des chercheurs britanniques (Merilänen et al., 2008).

Comme le soulignent Tietze et Ditz (2012) à propos d'une étude de Curry et Lillis (2004), comparant des données recueillies au Portugal, en Espagne, en Hongrie et en Slovaquie (2004) :

This normalized and exclusive use of the English language is a further illustration of hegemonic practices, through which meanings that « fall outside the dominant ideology "... become considerably harder (and riskier for individual careers) to express. English is, therefore, not an «innocent» system of syntax through which words and sentences are generated, rather it is a shaping influence on the very meaning of the texts produced (Tietze, 2004, pp. 9-10).

C'est ce processus intellectuel hégémonique qui alimente aussi la contestation et la résistance dont ce numéro spécial est un exemple concret. Mais, comme l'exemple finlandais cité plus haut le montre, tout comme d'autres débats récents observés à ce sujet dans le champ 
européen de langue anglaise (Czarniawska, 2006 ; Adler \& Harzing, 2009 ; Grey, 2010), cette contestation n'est pas uniquement l'apanage de pays latins.

Le champ francophone continue, quant à lui, à vouloir défendre, malgré certaines tendances à la standardisation, ses singularités intellectuelles et sociales ainsi que sa langue (Berry 2004 ; Hatchuel, 2004 ; Chanlat, $2014 \mathrm{a}$; Hagège, 2012 ; Bayart, Borzeix \& Dumez, 2010).

La quatrième touche l'émergence possible d'un champ latin. En tant qu'acteur dans le champ international des études organisationnelles, depuis plus de trente ans, ce qui me frappe, c'est la complicité très grande qui existe spontanément entre de nombreux chercheurs francophones, lusophones, hispanophones et italianophones. On observe ainsi que les travaux importants de langue française sont en général connus et traduits par les chercheurs lusophones et hispanophones, voire italianophones ; ils le sont peut-être moins par les Espagnols qui, comme nous le montre notre collègue, sont plus tournés pour des raisons historiques, notamment ceux et celles qui travaillent dans les écoles privés, vers l'Amérique du Nord.

Ce phénomène qui semble toutefois moins prégnant en Amérique latine a permis de maintenir en raison des liens historiques, notamment entre le Brésil et la France et le monde francophone, un dialogue par la traduction (Aquino-Alves \& Pozzebon, 2013). Le colloque que nous avons organisé nous-mêmes avec des collègues mexicains à Zacatecas au Mexique en 2000 sur ce sujet a clairement témoigné non seulement de ce lien mais aussi de cette complicité intellectuelle entre chercheurs latino-américains et francophones (Chanlat, Fachin, \& Fischer, 2005, 2006).

Pour ma part, c'est lors de mes premiers voyages au Brésil au début des années 90 que j'ai découvert les travaux de Alberto Guerreiro Ramos, de Mauricio Tragtenberg et ceux de Fernando Prestes Motta avec lequel j'ai entretenu une conversation à la fois intellectuelle et amicale jusqu'à sa disparition, et qui a eu la gentillesse de participer à l'effort collectif de traduction de mon ouvrage de référence, traduction organisée magnifiquement par Ofelia Torres (Chanlat, 1992, 1994, 1996) ; tout comme j'ai pris connaissance lors de mes séjours au Mexique des travaux du CIDE et de ceux du groupe des études organisationnelles de la UAM-Iztapalapa (Ibarra-Colorado, 2006).

Comme vient de le rappeler tout récemment le secrétaire perpétuel de l'Académie suédoise, Horace Engdahl, dans un entretien au journal Le Monde (2014, p. 10) : L'anglais est une langue importante, mais ce n'est pas la langue universelle. La seule langue universelle, c'est la traduction.

C'est ce qui, pour ma part et pour bon nombre d'autres collègues de par le monde, notamment latino-américains, nous a amenés à agir de la sorte. La traduction est au cœur de la diffusion des travaux et pas seulement en anglais. Les chercheurs de langue latine ont beaucoup à gagner à se fréquenter et à faire connaître les travaux importants aux uns et aux autres. L'obstacle linguistique étant par ailleurs moins dur à franchir. Le colloque de Zacatecas, tout comme ceux organisés régulièrement à Salvador par Tânia Fischer, en sont de belles illustrations (Chanlat, Fachin, \& Fischer, 2005 ; 2006).

Ces défis sont de deux ordres : le premier est d'ordre socioéconomique et concerne la production de la richesse et sa répartition.

Au cours des dernières décennies, la montée des inégalités (Piketty, 2013) nous montre que beaucoup de choses restent à faire pour y remédier, tant dans les pays du Nord que dans les pays du Sud ; les débats qui ont surgi au Brésil lors de l’organisation de la Coupe du monde en sont une vivante illustration. L'écart grandissant entre les pays et à l'intérieur des pays est un enjeu social et politique que les chercheurs en gestion et en études organisationnelles ne peuvent éluder. Car les modes de gestion et d'organisation ne sont pas sans lien avec ce que nous observons, et avec un autre défi, tout aussi essentiel : le défi du développement durable.

A l'heure où s'accumulent les rapports de scientifiques nous montrant l'état dégradée de notre planète, nous ne pouvons plus faire en effet l'impasse sur cette question car il en va de notre survie et de notre bien être à long terme. Le monde de la gestion et les modes de production et de consommation que nos sociétés privilégient, sont désormais remis en question. Nous, chercheurs en gestion, nous nous devons de répondre à ces défis; nos sociétés et nos organisations nous attendent à ce sujet. Pour ce faire, les expériences sociales qui cherchent à y répondre dans nos univers respectifs se doivent d'être mieux connues et partagées. En effet, un des principaux résultats de la recherche en gestion n'est-il pas d'avoir montré qu'il n'y avait pas justement de One best way?

Les humains étant des acteurs, producteurs de leur réalité, les possibles à ce sujet sont multiples. A nous de trouver par l'imagination sociale créatrice un mode de vie plus équilibré à la fois socialement et durablement. Le mode de vie, notamment nord-américain n'est plus soutenable à terme, tout comme d'ailleurs dans une moindre mesure celui des Européens. En revanche, les données socioéconomiques venant des pays moins développés nous indiquent qu'il y a encore beaucoup de choses à faire dans ces pays. Cela doit nous conduire à innover, tant dans le monde scientifico-technique que dans le monde social et politique, et à revoir nos cadres de pensée à la lumière de l'histoire et de l'anthropologie, comme nous y invitent plusieurs textes de ce numéro. Les réflexions des pays du Sud sont de ce point de vue, de nouveau, essentielles, pour décentrer le débat, encore trop euro-américain.

Face à un tel agenda sociopolitique, l'univers de la gestion et des études organisationnelles qui s'y rattachent doit désormais privilégier avant tout des recherches 
pertinentes socialement plutôt que des débats méthodologiques ou théoriques abstraits, comme on peut malheureusement trop souvent l'observer dans bien des revues contemporaines, et des perspectives critiques fondées sur de véritables données empiriques. Cette inflexion se fera d'autant plus si de telles recherches sont enracinées dans leur contexte local, national et régional, si elles mettent en lumières la manière dont l'expérience est vécue en situation par les acteurs concernés, les micro-situations, dont parle un des auteurs de ce numéro, et si elles font appel à des postures et des méthodes pluralistes et innovantes.

C'est un peu l'appel auquel nous convient ici, chacun à leur manière, les auteurs des textes présentés dans ce numéro. C'est une réponse sociopolitique au défi de l'hégémonie anglo-saxonne que connaît le champ de la gestion aujourd'hui, notamment dans les univers non anglophones. Un tel appel est d'autant plus actuel que les Etats-Unis sont dans un déclin relatif, et que nous assistons à la montée d'un monde multipolaire au sein duquel de nombreux pays en développement ont pris une place de plus en plus importante, voire centrale (Chine, Inde, Brésil, Mexique).

À la suite de cette brève réflexion, il me semble évident en définitive que l'objet de ce numéro concerne la question de la défense et de l'illustration des spécificités de nos champs et de nos langues. Comme le montre ce numéro spécial plurilingue, cette défense n'est pas un signe de fermeture; bien au contraire, c'est un témoignage de l'ouverture et de la vitalité collective qui nous anime.

Tout comme les coordonnateurs et les auteurs de ce numéro, je tiens donc à m'associer à cette défense d'une vision à la fois vivante, existentielle et ouverte de nos langues et de nos champs, et à réitérer que loin d'être un plaidoyer contre l'anglais et le champ anglo-américain, cette position se veut avant tout une défense de la diver- sité linguistique, synonyme à la fois de diversité intellectuelle et culturelle, diversité indispensables à l'ouverture vers d'autres possibles. C'est ainsi que nous pourrons faire face aux défis auxquels sont confrontés, de nos jours, chacune de nos sociétés et le monde dans son ensemble, en renouant par ailleurs avec une vieille idée de Mary Parker Follett : l'unité n'est pas synonyme d'uniformité.

\section{RÉFÉRENCES}

Adler, N et Harzing, A.W. (2009). When knowledge wins: Transcending the sense and nonsense of academic rankings. The Academy of Management Learning \& Education, 8(1), 72-95.

Alcadipani, R., \& Rosa, A. (2011). From global management to glocal management: Latin American perspectives as a counter-dominant management epistemology. Canadian Journal of Administrative Science, 28(4), 453-466.

Aquino, A., M. \& Pozzebon, M. (2013) How to resist linguistic domination and promote knowledge diversity, RAE-Revista de Administração de Empresas, 53(6), 629-633.

Bargiela-Chiappini, F. (2001). Management, culture and discourse in international business. In M. Stroinska (Ed.), Relative points of view. Linguistic representations of culture (144-160). Oxford, England: Berghahn Books.

Battilana, J., Anteby, M., \& Sengul, M. (2010). The circulation of ideas across academic communities: When locals re-import exported ideas. Organization Studies, 31(6), 695-713.

Bayart, D., Borzeix, A., \& Dumez, H. (Dir). (2010). Langage et organisations Sur les traces de Jacques Girin. Les Éditions de l'École polytechnique: Paris.

Bayley, J. \& Clegg, S. (Ed) (2007). International encyclopedia of organization studies. Thousand Oaks: Sage.

Berry, M. (2004). Vers un audimat des savants? Invitation au débat. Gérer et comprendre, (77), 99.

Bourdieu, P. (1982). Ce que parler veut dire. Paris: Fayard.

Bourdieu, P. (1987). Choses dites. Paris: Les Éditions de Minuit.

Chanlat, J-F. (Coord), (1992). O indivíduo na organização: Dimensões esquecidas, Vol. I, Sao Paulo: Éditeur Atlas.
Chanlat, J-F. (Coord). (1994). O indíviduo nas organizações: Dimensões esquecidas. Vol II, Sao Paulo: Éditeur Atlas

Chanlat, J-F. (Coord). (1996). O individuo nas organizações: Dimensões esquecidas. Vol III, Sao Paulo: Éditeur Atlas.

Chanlat, J-F., Fachin, R., \& Fischer, T (Dir). (2005). Analysisis das organizaçoes, Perspectivas latinas, Porto Alegre: Casa editorial da Universidade Federal do Rio Grande do Sul.

Chanlat, J-F., Fachin, R., \& Fischer, T (Dir). (2006). Analysisis das organizaçoes, Perspectivas latinas, Porto Alegre: Casa editorial da Universidade Federal do Rio Grande do Sul.

Chanlat, J-F. (2014a). Langue et pensée dans le champ de la recherche en gestion : Constats, enjeux et atouts de la langue française. Gérer et comprendre, (115), 4-17.

Chanlat, J-F. (2014b). Language and thinking in Organization Studies: The visibility of French OS production in the Anglo-Saxon OS field". International Journal of Organizational Analysis, $22(4), 504-533$.

Courpasson, D., Arellano-Gault, D., Brown, A., \& Lounsbury, M. (2008). Organization Studies on the look-out? Being read, being listened to. Organization Studies, 29(11), 1383-1390.

Curry, M. J., \& Lillis, T. (2004). Multilingual scholars and the imperative to publish in English: Negotiating interests, demands and rewards. TESOL Quarterly, 38(4), 663-688.

Czarniawska, B,. (2006). The quiet European? Journal of Management Inquiry, 15, 332-334.

Dameron, S. \& Durand, T. (Ed). (2008). The future of business schools. Londres: MacMillan.

Eraly, A, (2011). "Les enjeux de l'évaluation. Du discours aux pratiques», in SERVAIS (P.), L'évaluation de la recherche en sciences humaines et sociales, Louvain-la-Neuve (Belgique), Bruylant-Academia, 15-36.

Grey, C. (2010). Organization studies: Publications, politics and polemic. Organization Studies, 31(6), 677-694.

Ibarra-Colado, E. (2006). Organization studies and epistemic coloniality in Latin America: thinking otherness from the margins, Organization, 13(4), 463-488.

Hagège, C. (2012). Contre la pensée unique. Paris: Editions Odile Jacob.

Hatchuel, A. (2004). Les limites des métriques d'impact. Réponse à Vincent Mangematin. Gérer et comprendre, (77), 100-102.

Lillis, T., \& Curry, M. J. (2006). Professional academic writing by multilingual scholars: Interactions with literacy brokers in the production of English-medium texts. Written Communication, 23(1), 3-35.

Ljosland, R. (2007). English in Norwegian academia: A step towards diglossia? World Englishes, 26(4), 395-410. 
Lussier, S. (2014). Les enseignants-chercheurs en gestion à l'épreuve des nouvelles injonctions institutionnelles : une étude France-Québec. Thèse de doctorat en sciences de gestion, Ecole doctorale, Université Paris-Dauphine.

Meriläinen, S., Tienari, J., Thomas, R., \& Davies, A. (2008). Hegemonic academic practices: Experiences of publishing from the periphery. Organization, 15(4), 584-597.

Pesqueux, Y. \& Tyberghein, J-P. (2009). L'école japonaise d'organisation. Paris: Editions AFNOR.

Piketty, T. (2013). Le Capital au XXI siècle. Paris: Seuil.
Tietze, S. (2004). Spreading the management gospel in English. Language and Intercultural Communication, 4(3), 175-189.

Tietze, S. \& Dick, P. (2012). The victorious english language: Hegemonic practices in the management academy, Journal of Management Inquiry. Advance online publication. Retrieved from http://jmi.sagepub.com/content/early/2012/04/29/1056492612444316. full.pdf+html

Tsui, A. S. (2007). «From homogenization to pluralism: International management research in the academy and beyond». Academy of Management Journal, 50, 1353-1364.
Tsuda, Y. (2013). Speaking against the hegemony of english problems, ideologies and solutions. In The Handbook of Critical Intercultural Communication. Nakayama, T \& Halualani, R, T. London: Basil Blackwell, 248-269.

Üsdiken, B. (2010). Between contending perspectives and logics: Organizational studies in Europe. Organization Studies, 31(6), 715-735.

Willmott, H. (2011). Journal list fetishism and the perversion of scholarship: reactivity and the ABS list. Organization, 18(4), 429-441. 